Pacific Journal of Mathematics

ISOMETRIC ISOMORPHISMS OF MEASURE ALGEBRAS 


\section{ISOMETRIC ISOMORPHISMS OF MEASURE ALGEBRAS}

\section{ROBERT S. STRICHARTZ}

The following theorem is proved:

If $G_{1}$ and $G_{2}$ are locally compact groups, $A_{i}$ are algebras of finite regular Borel measures such that $L^{1}\left(G_{i}\right) \leqq A_{i} \cong \mathscr{L}\left(G_{i}\right)$ for $i=1,2$, and $T$ is an isometric algebra isomorphism of $A_{1}$ onto $A_{2}$, then there exists a homeomorphic isomorphism $\alpha$ of $G_{1}$ onto $G_{2}$ and a continuous character $\chi$ on $G_{1}$ such that $T \mu(f)=\mu(\chi(f \circ \alpha))$ for $\mu \in A_{1}$ and $f \in C_{0}\left(G_{2}\right)$.

This result was previously known for abelian groups and compact groups (Glicksberg) and when $A_{i}=L^{1}\left(G_{i}\right)$ (Wendel) where $T$ is only assumed to be a norm decreasing algebra isomorphism.

A corollary is that a locally compact group is determined by its measure algebra.

If $G$ is a locally compact group with left Haar measure $m$, then the Banach space $\mathscr{C}(G)$ of finite complex regular Borel measures (the dual of the Banach space $C_{0}(G)$ of all continuous functions vanishing at infinity on $G$ ) can be made into a Banach algebra by defining multiplication of two elements $\mu, \nu \in \mathscr{C}(G)$ to be convolution:

$$
\mu * \nu(f)=\iint f(s t) d \mu(s) d \nu(t) \quad \text { for } \quad f \in C_{0}(G) .
$$

The subspace $L^{1}(G)$ of all measures absolutely continuous with respect to $m$ is a closed two-sided ideal and hence a subalgebra.

In [1; Theorems 3.1 and 3.2] it is shown that if $G_{1}$ and $G_{2}$ are either both abelian or both compact, then any algebraic isomorphism $T$ of a subalgebra $A_{1}$ of $\mathscr{L}\left(G_{1}\right)$ containing $L^{1}\left(G_{1}\right)$ onto a subalgebra $A_{2}$ of $/ /\left(G_{2}\right)$ containing $L^{1}\left(G_{2}\right)$ which is norm-decreasing on $L^{1}\left(G_{1}\right)$ has the form

$$
T \mu(f)=\mu(\chi(f \circ \alpha)) \quad \mu \in A_{1} \quad f \in C_{0}\left(G_{2}\right)
$$

where $\alpha$ is a homeomorphic isomorphism of $G_{1}$ onto $G_{2}$ and $\chi$ is a character on $G_{1}$. In this note we shall prove that $(*)$ holds where $T$ is assumed to be an isometry but $G_{1}$ and $G_{2}$ may be arbitrary locally compact groups. Our starting point will be the theorem of Wendel [2; Theorem 1] that any isometric isomorphism $T: L^{1}\left(G_{1}\right) \rightarrow L^{1}\left(G_{2}\right)$ is of the form $(*)$.

THEOREM. If $G_{1}$ and $G_{2}$ are locally compact groups and $T$ is an isometric isomorphism of a subalgebra $A_{1}$ of $\mathscr{C}\left(G_{1}\right)$ containing $L^{1}\left(G_{1}\right)$

Received March 8, 1964. 
onto a subalgebra $A_{2}$ of $\mathscr{C}\left(G_{2}\right)$ containing $L^{1}\left(G_{2}\right)$ then $T$ has the form $(*)$. Conversely, the equation $(*)$ defines an isometric isomorphism of $\mathscr{L}\left(G_{1}\right)$ onto $\mathscr{L}\left(G_{2}\right)$ for every choice of $\alpha$ and $\chi$.

Lemma. ${ }^{1}$ Let $\mu, \nu \in \mathscr{L}(G)$. Then $\mu \perp \nu$ if and only if $\|\mu+\nu\|=$ $\|\mu-\nu\|=\|\mu\|+\|\nu\|$.

Proof. Suppose $\mu \perp \nu$. Then there exists a disjoint partition of $G$ into sets $A, B$ such that $|\mu|(B)=|\nu|(A)=0$. Thus

$$
\begin{aligned}
\|\mu \pm \nu\| & =|\mu \pm \nu|(G)=|\mu \pm \nu|(A)+|\mu \pm \nu|(B) \\
& =|\mu|(A)+|\nu|(B)=\|\mu||+\| \nu \| .
\end{aligned}
$$

Conversely, assme $\|\mu+\nu\|=\|\mu-\nu\|=\|\mu\|+\|\nu\|$. Let $\mu=$ $f \nu+\mu_{s}$ where $f \in L^{1}(\nu)$ and $\mu_{s} \perp \nu$ be the Lebesgue decomposition of $\mu$ with respect to $\nu$. Then

$$
\begin{aligned}
\|\mu \pm \nu\|=\|\mu\|+\|\nu\|=\| f \nu & +\mu_{s}\|+\| \nu \| \\
& =\|f \nu\|+\left\|\mu_{s}\right\|+\|\nu\| .
\end{aligned}
$$

But $\|\mu \pm \nu\|=\|(1 \pm f) \nu\|+\left\|\mu_{s}\right\|$ so $\|(1 \pm f) \nu\|=\|f \nu\|+\|\nu\|$. Thus $f=0$ a.e. with respect to $\nu$ hence $\mu \perp \nu$.

Proof of theorem. The converse is an easy verification. Let $T$ be an isometric isomorphism of $A_{1}$ onto $A_{2}$. We shall show first that $T$ maps $L^{1}\left(G_{1}\right)$ onto $L^{1}\left(G_{2}\right)$ and hence has the form $(*)$ when restricted to $L^{1}\left(G_{1}\right)$, and then that $(*)$ extends to all of $A_{1}$.

Indeed $L^{1}\left(G_{i}\right) \quad i=1,2$ will be shown to be the intersection of all nontrivial closed left ideals $I \subseteq A_{i}$ which satisfy

(**) $\mu \in I, \nu \in A_{i}$ and $\nu \perp \lambda$ whenever $\mu \perp \lambda$ and $\lambda \in A_{i}$ imply $\nu \in I$.

$T$ and $T^{-1}$ clearly preserve the property of being a closed left ideal and by the lemma they preserve $(* *)$. Thus $T$ maps $L^{1}\left(G_{1}\right)$ onto $L^{1}\left(G_{2}\right)$.

Now for $\mu \in L^{1}\left(G_{i}\right)$, the condition $\nu \in A_{i}$ and $\nu \perp \lambda$ whenever $\lambda \in A_{i}$ and $\mu \perp \lambda$ is equivalent to $\nu \ll \mu$. Clearly $\nu \ll \mu$ implies it, and conversely any $\nu$ satisfying it must be orthogonal to its singular part $\lambda$ in its Lebesgue decomposition $\nu=f \mu+\lambda$ with respect to $\mu$ since $\lambda \in A_{i}$. So $L^{1}\left(G_{i}\right)$ is a closed left ideal satisfying $(* *)$. Let $I \subseteq A_{i}$ be any nontrivial closed left ideal satisfying $(* *)$. Then $I$ must contain a nonzero $L^{1}$ measure since $\alpha * \mu \in L^{1}$ and is nonzero for $\mu \neq 0$ in $I$ and $\alpha$ is a suitable element in an $L^{1}$ approximate identity. The total variation of this measure is absolutely continuous with respect to it, hence in I. By convolving this with an appropriate $L^{1}$ approximation to a point

${ }^{1}$ I am indebted to George Reid for suggesting this lemma. 
mass, we get a measure $\nu \in I$ strictly positive in a neighborhood of the identity (the convolution of an $L^{1}$ and an $L^{\infty}$ function is continuous). But there is an $L^{1}$ approximate identity absolutely continuous with respect to $\nu$, hence in $I$. Since $I$ is a closed ideal, $L^{1} \cong I$.

Thus we have $(*)$ holding for all $\nu \in L^{1}\left(G_{1}\right)$. Let $\mu \in A_{1}$, and $\nu \in L^{1}\left(G_{1}\right)$. Then $\mu * \nu \in L^{1}\left(G_{1}\right)$ so

$$
\begin{aligned}
\iint f(\alpha(s t)) \chi(s t) d \mu(s) d \nu(t) & =T(\mu * \nu)(f)=(T \mu * T \nu)(f) \\
& =\iint \chi(t) f(r \alpha t) d T \mu(r) d \nu(t)
\end{aligned}
$$

so $(*)$ holds for $\mu$ and all functions in $C_{0}\left(G_{2}\right)$ of the form $\int f(r \alpha t) \chi(t) d \nu(t)$ where $f \in C_{0}\left(G_{2}\right)$ and $\nu \in L^{1}\left(G_{1}\right)$. This class of functions is dense in $C_{0}\left(G_{2}\right)$ since $\nu$ may be taken in an $L^{1}$ approximate identity. Thus $(*)$ holds for all $C_{0}\left(G_{2}\right)$ by continuity, which proves the theorem.

COROLLARY. A locally compact group is determined by its measure algebra.

This corollary was obtained independently by B. E. Johnson (Proc. Amer. Math. Soc. 1964). His results imply the main theorem under the hypothesis that each $A_{i}$ contains all point masses.

\section{BIBLIOGRAPHY}

1. Irving Glicksberg, Homomorphisms of certain algebras of measures, Pacific J. Math. 10 (1960), 167-191.

2. J. G. Wendel, On isometric isomorphism of groups algebras, Pacific J. Math. 1 (1951), 305-311.

PRINCETON UNIVERSITY 



\section{PACIFIC JOURNAL OF MATHEMATICS}

\section{EDITORS}

H. Samelson

Stanford University

Stanford, California

R. M. Blumenthal

University of Washington

Seattle, Washington 98105
J. Dugundu

University of Southern California Los Angeles, California 90007

*Richard Arens

University of California

Los Angeles, California 90024

\section{ASSOCIATE EDITORS}
E. F. BECKENBACH
B. H. NeumanN
F. WOLF
K. YOSIDA

\section{SUPPORTING INSTITUTIONS}

UNIVERSITY OF BRITISH COLUMBIA CALIFORNIA INSTITUTE OF TECHNOLOGY UNIVERSITY OF CALIFORNIA MONTANA STATE UNIVERSITY

UNIVERSITY OF NEVADA

NEW MEXICO STATE UNIVERSITY

OREGON STATE UNIVERSITY

UNIVERSITY OF OREGON

OSAKA UNIVERSITY

UNIVERSITY OF SOUTHERN CALIFORNIA
STANFORD UNIVERSITY

UNIVERSITY OF TOKYO

UNIVERSITY OF UTAH

WASHINGTON STATE UNIVERSITY

UNIVERSITY OF WASHINGTON

AMERICAN MATHEMATICAL SOCIETY CALIFORNIA RESEARCH CORPORATION SPACE TECHNOLOGY LABORATORIES NAVAL ORDNANCE TEST STATION 


\section{Pacific Journal of Mathematics}

\section{Vol. 15, No. $1 \quad$ September, 1965}

Donald Charles Benson, Unimodular solutions of infinite systems of linear

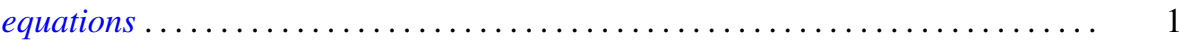

Richard Earl Block, Transitive groups of collineations on certain designs . . . . . . 13

Barry William Boehm, Existence of best rational Tchebycheff approximations .... . 19

Joseph Patrick Brannen, A note on Hausdorff's summation methods . . . . . . . . . . 29

Dennison Robert Brown, Topological semilattices on the two-cell ............ 35

Peter Southcott Bullen, Some inequalities for symmetric means . . . . . . . . . . 47

David Geoffrey Cantor, On arithmetic properties of coefficients of rational

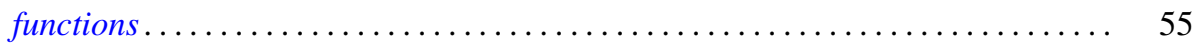

Luther Elic Claborn, Dedekind domains and rings of quotients . . . . . . . . . 59

Allan Clark, Homotopy commutativity and the Moore spectral sequence ........ 65

Allen Devinatz, The asymptotic nature of the solutions of certain linear systems of

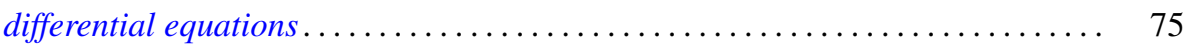

Robert E. Edwards, Approximation by convolutions ................... 85

Theodore William Gamelin, Decomposition theorems for Fredholm operators . . . . . 97

Edmond E. Granirer, On the invariant mean on topological semigroups and on

topological groups .................................. 107

Noel Justin Hicks, Closed vector fields . . . . . . . . . . . . . . . . . . . 141

Charles Ray Hobby and Ronald Pyke, Doubly stochastic operators obtained from

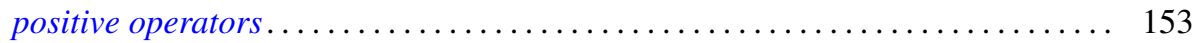

Robert Franklin Jolly, Concerning periodic subadditive functions . . . . . . . . . 159

Tosio Kato, Wave operators and unitary equivalence . . . . . . . . . . . . . . 171

Paul Katz and Ernst Gabor Straus, Infinite sums in algebraic structures . . . . . . . 181

Herbert Frederick Kreimer, Jr., On an extension of the Picard-Vessiot theory ...... 191

Radha Govinda Laha and Eugene Lukacs, On a linear form whose distribution is

identical with that of a monomial ......................... 207

Donald A. Ludwig, Singularities of superpositions of distributions . . . . . . . . . 215

Albert W. Marshall and Ingram Olkin, Norms and inequalities for condition

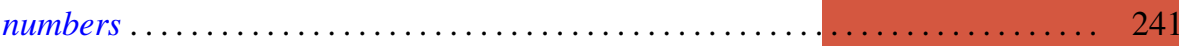

Horace Yomishi Mochizuki, Finitistic global dimension for rings . . . . . . . . . . 249

Robert Harvey Oehmke and Reuben Sandler, The collineation groups of division

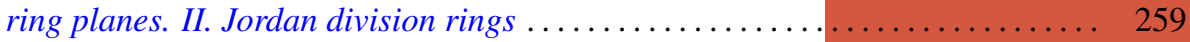

George H. Orland, On non-convex polyhedral surfaces in $E^{3} \ldots \ldots \ldots \ldots \ldots \ldots \ldots 267$

Theodore G. Ostrom, Collineation groups of semi-translation planes . . . . . . . . 273

Arthur Argyle Sagle, On anti-commutative algebras and general Lie triple

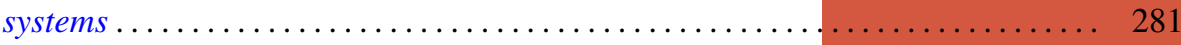

Laurent Siebenmann, A characterization of free projective planes . . . . . . . . . 293

Edward Silverman, Simple areas.................................. 299

James McLean Sloss, Chebyshev approximation to zero .................. 305

Robert S. Strichartz, Isometric isomorphisms of measure algebras . . . . . . . . . 315

Richard Joseph Turyn, Character sums and difference sets . . . . . . . . . . . . 319

L. E. Ward, Concerning Koch's theorem on the existence of arcs . . . . . . . . . . 347

Israel Zuckerman, A new measure of a partial differential field extension ......... 357 